\title{
Enfoque de derechos humanos en la política pública de discapacidad*
}

\author{
Carlos Parra Dussan ${ }^{* *}$ \\ María Teresa Palacios Sanabria ${ }^{* * *}$
}

\begin{abstract}
Resumen
La evolución del concepto de discapacidad ha pasado en los últimos años, de un enfoque biomédico a un enfoque de derechos humanos, éste considera los aspectos individuales de la persona con discapacidad en relación con un contexto social, cultural y físico. Las condiciones de interacción entre el individuo y el contexto, así definido, son las que determinan la magnitud de la discapacidad de un individuo su familia y la sociedad.

En el presente artículo, se examinan algunos temas que son objeto de debate, tales como la relación existente entre las políticas públicas de orden nacional y su grado de cumplimiento respecto de los estándares establecidos por la comunidad internacional. Así mismo se evalúa la conveniencia de manejar en el tema concreto de la discapacidad un enfoque orientado hacía la defensa de los derechos humanos y finalmente la recepción de estas tendencias en sede de las instancias locales.
\end{abstract}

Así pues, las realidades del contexto social, económico, político, al igual que los compromisos de carácter internacional adquiridos por Colombia, obligan realizar un esfuerzo de comprensión de las políticas públicas nacionales con las locales, las tendencias mundiales junto al desarrollo, la obligación del cumplimiento de convenios y tratados sobre derechos humanos y el derecho internacional humanitario, con el propósito de tener una visión más integral en el abordaje y solución a los problemas del sector de la discapacidad que afecta nuestro país.

El Plan Nacional de Intervención en Discapacidad está fundamentado en varios elementos conceptuales: tales como concepto de Manejo Social del Riesgo (MSR), teoría económica que fundamenta a su vez el nuevo enfoque de protección social a partir del cual se crea el Ministerio de la Protección Social.

En el tema de discapacidad, la discusión se centra por tanto en decidir si la construcción de la política se debe desarrollar con un enfoque de Manejo Social del Riesgo o con un enfoque de Derechos y de Participación Ciudadana más compatible con el esquema anterior al Conpes.

Fecha de recepción del artículo: 25 de octubre de 2007

Fecha de aprobación del artículo: 7 de noviembre de 2007

"Resultado del proyecto de investigación "Política pública de discapacidad en Colombia". Avalado y financiado por la Universidad del Rosario.

** Abogado Universidad del Rosario, Doctor en derechos fundamentales Universidad Carlos III de Madrid. Especialista derecho constitucional en el centro de estudios políticos y constitucionales de Madrid, Director línea de investigación grupos de especial protección constitucional. Facultad de jurisprudencia. Docente investigador Universidad del Rosario. Bogotá, Colombia. Coordinador red de universidades por la discapacidad.

${ }^{* * *}$ Abogada de la Universidad del Rosario, Especialista en Derechos Humanos, estudiante del doctorado de la Universidad de Sevilla, profesora de carrera académica de la facultad de jurisprudencia de la Universidad del Rosario 
De manera sucinta, el enfoque de derechos plantea que los individuos deben reconocerse, asumirse y ser considerados como sujetos de derechos fundamentales inalienables e irrenunciables. El desarrollo está en este sentido orientado hacia los intereses del pueblo, a partir de procesos de gerencia y gestión social en los que las comunidades tienen el poder de determinar los derroteros para su progreso.

Se considera que el derecho internacional de los derechos humanos constituye un marco conceptual aceptado por la comunidad internacional, capaz de orientar el proceso de formulación, implementación y evaluación de políticas en el campo del desarrollo. Del mismo modo se constituye como una guía para la cooperación y la asistencia internacional respecto a las obligaciones de los gobiernos con respecto a las personas con discapacidad.

En términos de los derechos humanos y el derecho internacional humanitario, el país ha suscrito una serie de convenios y tratados internacionales, como la Convención Interamericana para la Eliminación de Todas las Formas de Discriminación contra las Personas con Discapacidad, que obligan al Estado a la adopción de una serie de decisiones políticas, administrativas y jurídicas que permitan la efectiva protección a los derechos humanos de esta población.

\title{
Palabras clave
}

Política pública, derechos humanos, discapacidad, enfoques, pobreza y exclusión.

\begin{abstract}
The evolution of the concept of disability has moved from a biomedical focus to a human rights focus in the last years. The human rights focus regards people with disabilities' individual features with relation to their social, cultural y physical context. The interaction conditions between people and their context determines someone's disability magnitude, as well as their family's and the society's disability magnitude.

This article will examine some issues object of debate among those who intent to build relationships between public policies, national and international development strategies and human rights international law. Besides, it will analyze some points of view about the pertinence of a human rights focus in Colombia social, cultural and political context.

The social, economic and cultural context and Colombian international commitments press on to make an effort to connect local and national policies, global tendencies of development and compliance with human rights and humanitarian law in order to achieve a more comprehensive vision. It will allow society to confront and solve disability problems in a better way.

The Disability Intervention National Program has its grounds in the following conceptual elements: Risk Social Management (RSM), which refers to the economic theory that supports a social protection focus. This last focus is the creation base of the Social Protection Office.

In the field of disability, the discussion's main question is whether policies should be build from a RMS focus or from a civilian participation focus, which is more compatible with the approach previous the Conpes document.
\end{abstract}


In short, the human rights focus maintains individuals should be recognized, assumed and regarded as fundamental rights owners. Therefore, this focus' development should be guided by people's interests by means of social management processes due to the fact they allow communities to participate and take decisions about their own development.

Human rights international law is a conceptual framework accepted by the international community, which is able to guide policies' design, establishment and testing in the field of development. Furthermore it can be a guideline for international assistance and cooperation related to people with disabilities.

Colombia has signed some human rights and humanitarian treaties retated to disability, such as the Interamerican Convention on the elimination of all Forms of Discrimination against People with Disabilities, which constrains the estate to adopt administrative, political and judicial measures that encourage the effective protection of people with disabilities' rights.

\section{Key words}

Public politics, human rights, disability, focus, poverty, exclusion.

\section{Problema de investigación y método}

\section{Introducción}

Las políticas internacionales de occidente, la globalización de los mercados y los fondos internacionales inciden en el nuevo papel del Estado, que comienza a prefigurarse como de tipo neorregulador y con atributos de eficiencia, el cual espera dejar atrás las modalidades propias del Estado intervencionista y benefactor. En este contexto muta la relación entre economía y política: se pasa del papel predominante del Estado en la economía sobre todo en las políticas de desarrollo a una mayor influencia del sector privado, del mercado y de la financiación externa. Además, se pretende: que en las relaciones entre Estado y ciudadano se opere con la misma lógica de eficiencia, como lo hace el sector privado; que se abandone la intervención en áreas de la producción directa y se permita que las reglas del mercado regulen la economía. Para esto, se plantea la necesidad de privatizar algunas esferas de la acción estatal, eliminar algunas instituciones estatales y modernizar otras para hacer que su acción sea más eficiente.

Tradicionalmente la lógica de la política pública estaba fuertemente marcada por el presupuesto que el Estado debería regular el mercado. Era aquélla de la acción propia de un ente intervencionista, concebido como el actor fundamental del desarrollo. Dentro de esta lógica, el ente institucional formulaba las políticas públicas y su implementación, en lo fundamental, era igualmente de su competencia; por lo tanto, se esperaba que satisficiera las condiciones básicas de vida de aquella parte de la sociedad, así como los servicios públicos gratuitos o subsidiados, la salud, la educación, entre otros. Quizá porque al considerarlo como regulador de los medios de producción, esto le permitía garantizar los recursos destinados a la financiación de los servicios básicos de la población menos favorecida.

En el escenario hegemónico del neoliberalismo, parece haberse invertido la lógica como resultado del desplazamiento, en términos de quién es, del actor fundamental del desarrollo. Hoy en día se considera que este se sitúa en la sociedad civil y que son los actores privados, junto con el mercado, quienes pueden estimular los procesos de desarrollo. Son los postulados del Estado mínimo entendido como paradigma de los discursos denominados neoliberales o neoclásicos. Sin embargo, en la medida en que el período de auge extremo de políticas neoliberales 
ha hecho crisis al mostrar sus fisuras por los distintos polos, se le buscan nuevas funciones al Estado: Regular una forma nueva y exigir eficiencia en todos los procesos.

Los especialistas latinoamericanos han dado en llamarlo neoestructuralismo, para referirse a los esfuerzos de relectura del estructuralismo cepalino esta redefición del concepto conlleva cambios por lo menos en los siguientes aspectos:

- La concepción de la apertura, que pasa de una ideación indiscriminada a una basada en la selectividad, la reciprocidad y el relieve en lo sectorial.

- El rol del Estado, que pasa de defender el mercado per se y lo considera entonces como el principal dinamizador del desarrollo. Éste debe dar la dirección estratégica de la economía.

- La política económica, hace por tanto más hincapié en los aspectos fiscales y minimiza lo monetario-financiero.

Desde este nuevo concepto de Estado, las políticas públicas, en sus nociones básicas, sufren una serie de modificaciones a lo largo del documento. Por ejemplo, en el protagonismo de la sociedad civil y de las organizaciones privadas, en su formulación e implementación y en la incertidumbre de la sociedad civil de poder compartir su nuevo rol con un Estado mínimo con pocos recursos, que garantice un verdadero impacto social respecto a la implementación de la política, a tal punto que la actual crisis no sólo colombiana, sino latinoamericana puede afectar los logros en los procesos de integración obtenidos por esta población a partir de un modelo asistencial básico.

\section{Elementos de la política pública en discapacidad}

En la actualidad, en el sector de la discapacidad, es vigente la reflexión sobre la definición de qué es Política Pública, pues el Plan Indicativo 2003-2006 se había presentado como la Política Pública en Discapacidad con unos desarrollos, como ya se dijo, más bien sectoriales y de Gobierno, y con alguna intervención de la sociedad civil y el sector privado.

El Conpes 80 de 2004 aparece, en un principio, como una nueva política pública, pero pronto se hace claridad que se trata solo de un plan de gobierno.

Dentro de un escenario de fortalecimiento territorial, es importante hacer algunos aportes teóricos de definiciones de política pública como "... el conjunto coherente de principios, objetivos, estrategias y planes de acción que identifican, comprenden y abordan las problemáticas de una sociedad (económicas, políticas, sociales, culturales o ambientales) o condiciones de un grupo poblacional o comunidad, con el fin de darles solución o mejorar las condiciones de vida a partir de la acción colectiva, en el marco de un proyecto democrático de sociedad" (Sarmiento, 2005). O como "...un conjunto de decisiones que se traducen en acciones, estratégicamente seleccionadas (dentro de un conjunto de alternativas, conforme a una jerarquía de valores y preferencia de los interesados). Su dimensión es pública por el tamaño del agregado social sobre el cual inciden, pero sobre todo por su carácter imperativo, siendo éste revestido de la autoridad legítima y soberana del poder público" (Gracas en Guerrero, 2002).

Estos enfoques, ponen acento en la construcción local de la política pública, con un componente de participación activa de la sociedad civil organizada que ejerce sus plenos derechos; en acciones de consenso entre esta, el sector público y el privado como proceso legitimador de la política en el respeto por los procesos locales, que suponen una construcción de la misma en una dinámica descendente -de abajo hacia arriba- dejando de lado aspectos relacionados con la descentralizacón.

Así planteado, se deduce entonces que la política pública de discapacidad es un proceso dinámico en construcción. En este sentido, un 
plan de acción, como el presente ${ }^{1}$, solo se enfoca en unos lineamientos que da el gobierno para la construcción de política en los territorios para fortalecer las instituciones través de acciones específicas sectoriales y, en lo posible, intersectoriales. De esta manera, los desarrollos locales en el tema se facilitan. De ello se desprenden varios procesos. En primer lugar, el plan de gobierno propuesto debe pasar aún por una concertación conceptual a la luz de un bagaje amplio de enfoques teóricos y por la priorización de acciones que garanticen el fortalecimiento de los procesos locales y finalmente sostener la estructuración de estos respecto del nivel nacional.

\section{Los modelos de política pública en discapacidad}

El Plan Nacional de Intervención en Discapacidad está fundamentado en varios elementos conceptuales. Por un lado, el concepto de Manejo Social del Riesgo (MSR), teoría económica que fundamenta a su vez el nuevo enfoque de protección social con base en el cual se crea el Ministerio de la Protección Social.

Debe tenerse en cuenta que el concepto de discapacidad ha tenido transformaciones en los últimos tiempos, y ello se evidencia en la necesidad de superar el enfoque biomédico del mismo hacia la búsqueda de uno ecológico y de integración social, en el cual se entran a considerar aspectos individuales de la persona que padece una limitación y su relación con el contexto social cultural y físico.

Las condiciones de interacción entre el individuo y el contexto así definido, son las que determinan la magnitud de la discapacidad de un individuo y su familia. ${ }^{2}$

La política pública de discapacidad se fundamenta en los conceptos que se han citado, por ello, las acciones realizadas bajo el título de política pública apuntan a mejorar no solo la condición de salud de la persona con discapacidad, sino a incidir sobre ese entorno que la determina, es decir, a proporcionarle una atención integral.

En un primer momento, bajo la concepción de discapacidad definida por la nueva $\mathrm{CIF}^{3}$, se planteó una política pública que se definió como participativa, equitativa, descentralizada, solidaria, integral y concertada. Esta se tradujo en un plan indicativo, que desarrolló acciones sectoriales, cuyos objetivos principales apuntaban a la promoción de entornos saludables, la accesibilidad (vista como equiparación de Oportunidades) y la entrada a la habilitación/rehabilitación (Presidencia de la República, 2002).

A la luz de estos criterios, se trabajaron dentro de los Grupos de Enlace Sectorial (GES) los proyectos y tareas consignados en el plan indicativo $2003-2006$, no sin algunas dificultades operativas y conceptuales que surgieron con el desarrollo de acciones en los territorios.

Aunque la propuesta contenida en el libro "Bases para la formación de Política Pública" parte del marco teórico de Manejo Social del Riesgo, los componentes y líneas de acción que define (Prevención y Promoción, Equiparación de Oportunidades y Habilitación/Rehabilitación), responden más a la evolución del concepto de discapacidad que al enfoque de Manejo Social del Riesgo (MSR). En el momento en que el gobierno nacional emite el Conpes 080 de 2004, fundamentado específicamente en el MSR, los actores institucionales y de la sociedad civil con compromiso de trabajo en el tema de discapacidad, generan un debate entre defensores y detractores de los dos enfoques. Debate que aún no solo no termina, sino que empieza a ampliarse a otros aspectos teóricos relacionados con comprensión de lo que es la política pública.

\section{Enfoque de manejo social del riesgo MSR.}

El Manejo Social del Riesgo deriva en una propuesta de protección social que privilegia dos tipos de intervenciones públicas: 
- Asistir a las personas, hogares y comunidades para mejorar su manejo social del riesgo.

- Proporcionar apoyo a quienes se encuentran en extrema pobreza.

La primera intervención se centra en el desarrollo de estrategias de manejo del riesgo, clasificadas en estrategias de Reducción, Mitigación y Superación del riesgo. En esta actuación no solo el sector público aporta, sino también el sector privado y la sociedad civil, evidenciando una priorización de las poblaciones más vulnerables.

El Estado asume un papel de garante de la seguridad social de la población, pero transfiere el mercado de seguros al sector privado y una lógica de solidaridad, expresada en un subsidio por parte de los que pagan a los que no pueden pagar, como aporte de la sociedad civil.

La segunda intervención constituye un proceso de atención urgente de grupos poblacionales en situación crítica. Esto significa que el Estado destina una importante cantidad de recursos a atender a la población más vulnerable en situación crítica. Este enfoque que ha tenido desarrollos exitosos, pero, por su misma esencia, ha constituido programas de corto plazo que suponen una superación más o menos rápida de la situación de crisis. Esto no es siempre congruente con la realidad.

La protección social se concibe por tanto como asistencia social sumada a la seguridad social y al mercado laboral. Sin embargo, así vista, la protección social está fuertemente ligada al trabajo formal, esto plantea de entrada una dificultad dentro de una situación relacionada con la austeridad en el gasto público y los procesos de reestructuración del estado privilegian el empleo no formal e informal.

Los detractores de este modelo lo acusan de tender además hacia la privatización de la protección social y de tener un corte de carácter asistencial.

\section{El enfoque de derechos en discapacidad}

De manera sucinta, el enfoque de derechos plantea que los individuos deben reconocerse, asumirse y ser considerados como sujetos de derechos fundamentales inalienables e irrenunciables. El desarrollo está orientado hacia los intereses del pueblo, a partir de procesos de gerencia y gestión social en los que las comunidades tienen el poder de definir y decidir sus derroteros para su desarrollo.

En el tema de discapacidad, la discusión se centra en decidir si la construcción de la política se debe desarrollar con un enfoque de Manejo Social del Riesgo o con un enfoque de derechos y de participación ciudadana más compatible con el esquema anterior al Conpes.

El primero supone unas acciones del Estado tendientes a asegurar a la población con discapacidad contra posibles riesgos y el segundo implica que esta entra a participar activamente en la definición de la Política y en la toma de decisiones frente al tema de la discapacidad.

\section{El enfoque de derechos en las politicas y estrategias de desarrollo}

Se considera que el derecho internacional sobre los derechos humanos constituye un marco conceptual aceptado por la comunidad internacional, el cual es capaz de orientar el proceso de formulación, implementación y evaluación de políticas en el campo del desarrollo, lo que permite que se tenga como una guía para la cooperación y la asistencia internacional. Ello evidencia la necesidad de establecer claridad respecto a las obligaciones de los gobiernos donantes y receptores, el alcance de la participación social y los mecanismos de control y responsabilidad que se necesitan a nivel local e internacional.

Se analizan algunos puntos de encuentro y desencuentro entre el ámbito del desarrollo y el de los derechos humanos. Del mismo modo se 
procura establecer algunas relaciones entre ciertos derechos fundamentales -como el derecho a la igualdad, a la participación política y al acceso a la justicia-y las nociones de inclusión, participación y rendición de cuentas, usadas con frecuencia en las estrategias de desarrollo.

Por otro lado se examinan algunos temas que son objeto de debate en el intento por relacionar las políticas y estrategias nacionales e internacionales de desarrollo y el derecho internacional sobre derechos humanos. Finalmente expresa algunos puntos de vista sobre la pertinencia de este enfoque en el contexto político, social e institucional de Colombia .

El denominado "enfoque de derechos en las políticas y estrategias de desarrollo" considera el derecho internacional sobre derechos humanos como un marco conceptual que es aceptado por la comunidad internacional y que puede ofrecer un sistema coherente de principios y reglas en el ámbito del desarrollo. También lo trata como una guía amplia acerca de la forma de llevar a cabo el proceso construcción de las políticas al interior de los Estados. De otra parte constituye un antecedente del enfoque de la discapacidad en el ámbito del desarrollo, promovido desde el decenio de la discapacidad 2006-2016 por las agencias de cooperación y los organismos multilaterales de cooperación y asistencia. La participación social en ese proceso supone por tanto la supervisión de las obligaciones de los gobiernos donantes y en líneas generales, este tipo de enfoque basado en derechos se considera como el primer paso para otorgar poder a los sectores excluidos es reconocer que ellos son titulares de derechos que obligan al Estado.

Al introducir este concepto se procura cambiar la lógica de los procesos de elaboración de políticas, para que el punto de partida no sea la existencia de personas con necesidades que deben ser asistidas, sino sujetos que tengan el derecho a demandar determinadas prestaciones y conductas. Las acciones que se emprendan en este campo no son consideradas solo como el cumplimiento de mandatos morales o políticos, sino como la vía escogida para hacer efectivas las obligaciones jurídicas, imperativas y exigibles, impuestas por los tratados de derechos humanos. Los derechos demandan obligaciones y las obligaciones requieren mecanismos para hacerlas exigibles y darles cumplimiento.

Aunque los diversos marcos conceptuales sobre el enfoque de derechos parten de distintos fundamentos políticos y filosóficos, e incluso difieren a veces en las definiciones de pobreza, comparten la idea de que las situaciones de pobreza determinan que haya privación de algunas libertades básicas, positivas y también negativas, la libertad de evitar el hambre, la enfermedad y el analfabetismo; que la pobreza dependa de factores tanto económicos como culturales, sociales, legales y políticos.

Si bien la pobreza está relacionada con la insuficiencia de recursos económicos (de ingresos personales, por ejemplo) esto no significa forzosamente que los factores económicos sean los principales causantes de la pobreza. Ciertas prácticas culturales y algunos marcos políticos y jurídicos que facilitan o promueven la discriminación contra ciertos individuos o grupos, como las personas con discapacidad, actúan como mecanismos de exclusión social que causan o contribuyen a causar pobreza.

Los diversos marcos conceptuales no abordan todos los derechos, sino que procuran identificar aquellos que son fundamentales para las estrategias de desarrollo o de reducción de la pobreza, por tener relación constitutiva o instrumental con la pobreza. Así, por ejemplo, en ACNUDH (2004) se señalan tres diferentes formas de pertinencia de los derechos humanos en esas estrategias: la pertinencia constitutiva, la instrumental y la restrictiva respecto del contenido y el ámbito de las estrategias. Algunos derechos tienen pertinencia constitutiva cuando corresponden a capacidades consideradas básicas por la sociedad en cuestión y no se les da cumplimiento por insuficiencia de recursos 
económicos (por ejemplo, el derecho a la alimentación o el derecho a la salud). Otros derechos, como algunos civiles y políticos, tienen pertinencia instrumental porque contribuyen a evitar procesos sociales o políticos que pueden conducir a situaciones de pobreza. Así, la libertad de expresión y los derechos vinculados con el funcionamiento de democracias representativas, con elecciones periódicas y limpias, acotan las posibilidades de que la sociedad tolere situaciones de pobreza extrema (hambrunas, por ejemplo) sin exigir respuestas al gobierno o activar mecanismos para hacer efectiva la responsabilidad social o política.

Existen también derechos que tienen pertinencia instrumental, porque facilitan procesos sociales de consulta y evaluación que son indispensables para definir políticas o estrategias destinadas a reducir la pobreza: se trata de los derechos de participación, de información y de asociación o reunión. Por último, ciertos derechos tienen utilidad para las estrategias cuando pueden restringir o limitar los tipos de acciones permisibles. Así, por ejemplo, aunque sería totalmente razonable que un país muy poblado pero con escasos recursos quisiera adoptar medidas de control demográfico como parte de su estrategia para reducir la pobreza, no sería admisible que adoptara medidas como la esterilización forzosa, que viola la integridad física y la intimidad de las personas. De tal modo, ciertos derechos, cuyo incumplimiento no es en sí causa de pobreza y cuya promoción tal vez carezca de valor instrumental para las estrategias de desarrollo y reducción de la pobreza, pueden tener algún influjo en la orientación de las políticas al excluir ciertas opciones de intervención estatal por considerarse legalmente prohibidas.

Al respecto, un elemento común de los diversos marcos conceptuales es haber adoptado el principio de interdependencia de los derechos civiles y políticos, de los derechos económicos, sociales y culturales, aunque ciertamente no todos los marcos ponen el mismo énfasis en las diferentes categorías de derechos. Los derechos que han de considerarse, así como las prioridades, se definen por lo general en función de las diversas estrategias de desarrollo y sin atribuir a priori la preeminencia de alguna categoría de derechos por sobre otra. Contribuye a esta visión el examen de un núcleo de obligaciones negativas y positivas comunes a todas las categorías de derechos, que se agrupan en tres niveles: obligaciones de respetar, de proteger y de cumplir.

Los marcos conceptuales definen de partida, con mayor o menor detalle, las principales obligaciones que derivan de los derechos seleccionados como pertinentes a la estrategia de que se trate. Sobre la base de esas obligaciones fijan posibles metas e indicadores de desarrollo, como referencia para las metas e indicadores que habría que establecer en cada proceso participativo a nivel local.

Paradójicamente el campo de las del Alto Comisionado de las Naciones Unidas para los Derechos Humanos incluye claramente obligaciones, metas e indicadores para los derechos económicos, sociales y culturales pese a compartir la preocupación por muchos temas comunes, en particular referidos a la pobreza y a la exclusión, con organismos como UNICEF y algunos otros que conllevan a la correcta constitución de la democracia y su desarrollo.

El marco conceptual del DFID, por otro lado, si bien menciona con frecuencia derechos económicos, sociales y culturales, se organiza en torno a tres ideas centrales: inclusión, participación y cumplimiento de obligaciones, sin definir la estrategia en función de derechos en particular. Los derechos referidos se plantean en relación a cada uno de estos tres conceptos.

Las políticas de desarrollo y de los derechos humanos han ido por carriles paralelos, con pocos puntos de encuentro o conexión. Muchos de los conceptos habituales en el lenguaje del desarrollo, como la reducción de la pobreza, la participación, la inclusión, el buen gobierno y la responsabilidad y rendición de cuentas (accountability), se 
refieren a los mismos temas que en el campo de los derechos son descritos como el alcance de los derechos a la salud, a la alimentación, a la educación, a la libertad de expresión, a la participación política, a la igualdad y a la no discriminación y al acceso a la justicia, entre otros. En ocasiones, el lenguaje de los derechos ha sido considerado excesivamente político y poco neutral por algunas de las agencias de cooperación, e incluso es vetado por los mandatos de algunas instituciones financieras internacionales, como el Banco Mundial; sin embargo, esto no ha impedido que la agenda de esas instituciones aborde problemas de pobreza o de calidad institucional directamente vinculados con aquellos derechos.

Algunas críticas a la posibilidad de que las políticas de desarrollo asuman una lógica basada en los derechos cuestionan sobre todo la ambigüedad del contenido de las obligaciones que emanan de los derechos económicos, sociales y culturales. Además ponen en duda que estos derechos puedan ser exigibles en igual grado que los derechos civiles y políticos. También plantean que un enfoque de las políticas públicas basado en derechos puede resultar a veces demasiado rígido y, por ende, acotar de manera poco conveniente la discrecionalidad de quienes formulan las estrategias de desarrollo. Sin embargo, antes cabe decir que hay un argumento de peso tras esas objeciones, pues la relación entre los derechos humanos -en especial los económicos, sociales y culturales-y las políticas públicas es una cuestión espinosa, que no cuenta aún con elaboraciones suficientemente sólidas y coherentes, ni en el derecho internacional, ni en el derecho constitucional de los países latinoamericanos.

Los derechos no dicen mucho acerca del contenido de las políticas, pero sí pueden decir algo sobre la orientación general de ellas y brindar un marco conceptual que guíe su formulación e implementación. Los partidarios del enfoque basado en los derechos entienden que el cambio de perspectiva no implica modificaciones radicales o abruptas en las prácticas que las agencias de cooperación vienen realizando en los últimos años, pues rescatan los puntos de encuentro y sinergia entre el ámbito del desarrollo $\mathrm{y}$ el de los derechos humanos. Sostienen que las obligaciones que imponen los tratados de derechos humanos no son exorbitantes, ni le restan espacio a las políticas, sino que ponen de relieve las acciones mínimas que el Estado debe realizar. Además, postulan que los principales aportes que hace el enfoque basado en derechos a las estrategias de desarrollo son la vinculación de los derechos con la entrega de poder a los sectores empobrecidos y el fortalecimiento de los mecanismos de responsabilidad mediante el uso de la "infraestructura institucional" internacional y nacional existente, en el ámbito de los derechos humanos. Consideran también que los tratados de derechos humanos y su interpretación por los órganos internacionales brindan un marco explícito, claro, reconocido por todos los países y dotado de una fuerte legitimidad social y política. Indudablemente se mejorará la eficacia de las estrategias de desarrollo y la posibilidad de articular a actores estatales y no estatales en el escenario local, nacional e internacional.

Es evidente que la adopción de este enfoque en las estrategias de desarrollo latinoamericanas podría contar con una sólida infraestructura. En la región, el concepto de derechos humanos surgió como un medio para imponer límites a formas abusivas de uso del poder por el Estado, como un decálogo de aquellas conductas que el Estado no debería tener. No torturar, no privar arbitrariamente de la vida, no entrometerse en la vida privada y familiar de las personas, no discriminar. Esta concepción estuvo signada por la resistencia a las dictaduras militares en el Cono Sur en la década de 1970 y en Centroamérica en la década de 1980.

En los últimos años, el cuerpo de principios, reglas y estándares que componen el derecho internacional sobre los derechos humanos ha fijado con mayor claridad no solo las obligaciones negativas del Estado, sino también un cúmulo de obligaciones positivas. Esto significa que ha 
definido con mayor precisión no solo aquello que el Estado no debe hacer, a fin de evitar violaciones, sino también lo que debe hacer para lograr la plena materialización de los derechos civiles y políticos. Cuentan también los económicos, sociales y culturales. En tal sentido, los derechos humanos no son pensados hoy tan solo como un límite a la opresión y al autoritarismo, sino como un programa que puede guiar u orientar las políticas públicas de los Estados y contribuir al fortalecimiento de las instituciones democráticas, particularmente en procesos de transición o en democracias deficitarias o débiles.

En los países latinoamericanos, muchas organizaciones de derechos humanos, además de realizar una férrea fiscalización de las acciones estatales, han iniciado diálogos fructíferos con los gobiernos tendientes a incidir en la orientación de sus políticas y lograr un mejor funcionamiento de las instituciones públicas. Este cambio de perspectiva apunta a sumar, a la tradicional tarea de denunciar violaciones masivas o sistemáticas de derechos, una acción preventiva y de promoción capaz de evitar tales violaciones. En igual sentido, los órganos de supervisión internacional de derechos humanos, tanto a nivel universal como regional, han procurado no solo entregar reparaciones a las víctimas en casos particulares, sino también establecer un cuerpo de principios y estándares con el propósito de incidir en la calidad de los procesos democráticos y en los esfuerzos por llegar a tener sociedades más igualitarias e integradas.

Esta agenda de fortalecimiento institucional no ha estado siempre presente en el trabajo de supervisión internacional del Sistema Interamericano de Protección y Promoción de los Derechos Humanos, orientado a veces de manera exclusiva a ser el último recurso de justicia para víctimas de situaciones de violación masiva y sistemática de sus derechos. Sin embargo, dicho sistema cuenta con herramientas de intervención que permiten mejorar apreciablemente su aporte conceptual a los procesos de formulación de políticas En la práctica esto implica una meto- dología de trabajo distinta, en la que los niveles nacionales y departamentales se constituyen en otros de apoyo y orientación de los locales y no como estadios que señalan directrices de acción.

\section{Política pública y discapacidad}

Para el análisis y abordaje de las políticas públicas en nuestro país es importante establecer, basándose en los elementos expuestos, un marco de referencia que determina su origen, diseño y formulación (Vargas, 1995, pp. 55-65).

Teniendo en cuenta que toda acción estatal posible tiene un referente en términos de un futuro deseado de la situación problemática hacia la cual se espera que se desplace la política pública, el Estado - en representación de los ministerios de Salud, Trabajo, Educación, Comunicaciones, e instituciones adscritas como Inci e Insor y con la participación de la sociedad civil organizada- ha determinado como punto crítico en el contexto general de los problemas de la población con discapacidad, la falta o insuficiencia de atención integral y potenciación de la coordinación intersectorial e interinstitucional como estrategia coherente en el abordaje de los problemas derivados de la discapacidad. De esta manera garantizar, asimismo, la más adecuada utilización de los escasos recursos disponibles (Presidencia de la República, 2002).

Este problema combinó dos aspectos fundamentales. Por una parte, la visión del individuo no como ser integral, sino como sujeto de los servicios a partir de la estructura del Ejecutivo, constituida por sectores como educación, salud, trabajo, etc. Dicha circunstancia, sin embargo, no resolvió las necesidades de la persona con discapacidad a la luz de la integración, como su participación en los procesos sociales, políticos, culturales, entre otros.

Por otra, se establece la inconsistencia en las acciones conjuntas de los entes públicos que en su conjunto deberían orientar los esfuerzos a la solución de las necesidades de un grupo de 
personas. Debido a que por sus características determinadas, constituye el colectivo claramente identificado por el Estado, que es la población con discapacidad. Asimismo, se determina en el problema y se refuerza la falencia en forma consciente desde el Estado, al aceptar la falta de coordinación institucional, y además expresar la escasez de recursos para la ejecución de los programas o proyectos.

En el proceso de elección de la política pública, en el marco decisorio de un conjunto de prioridades establecidas para la solución del problema, el gobierno nacional, basándose en los resultados consolidados del trabajo realizado por las mesas regionales, dispuso la elección de las políticas para el caso de la discapacidad. Pero no se centró en el análisis de las alternativas, sino en la selección de las situaciones problemáticas de mayor peso, pues la metodología utilizada la relaciona en orden jerárquico según la gravedad en el déficit de los servicios.

En tal sentido, se determinó que la política de atención integral a las personas con discapacidad debería considerar, por lo tanto, los aspectos de prevención sanitaria y social y al igual que la educación para la salud; la detección y atención temprana y la protección específica; el diagnóstico y valoración de las situaciones de discapacidad; la atención de la salud; la educación; la rehabilitación profesional (incluso ocupacional); así como la habilitación y rehabilitación social. Lo anterior debe comprender el apoyo a la familia, la protección económica, la mejora de la accesibilidad física del entorno, la eliminación de las barreras arquitectónicas y de comunicación, la disponibilidad de transporte adecuado, las prestaciones técnicas. En este sentido debe contemplar el acceso al deporte, la cultura y el ocio, con el objetivo final de lograr la participación social plena y la vida independiente de las personas con discapacidad, en un contexto de normalización e integración.

Por cuanto las decisiones anteriores deben materializarse en términos de acciones (ya sean planes, programas, proyectos o acciones puntuales), la administración hizo referencia a los programas en el documento Plan Nacional de Atención para las Personas con Discapacidad, los programas, los subprogramas y los proyectos de inversión de acuerdo con los registros que cada una de las instituciones intervinientes tenían registrados para el año de 1997 en el Departamento Nacional de Planeación (DNP). Éstos fueron diseñados en vigencias anteriores y, por lo tanto, según la política, deberían ser valorados y, dado el caso, reformulados a la luz de la solución de la situación problemática establecida, al igual que las continuas reformas del Estado que particularmente se dieron en el período 1998-2002, en el orden nacional. Estas últimas obedecieron a orientaciones de política internacional relacionadas con la reforma del Estado y que se reflejaron en el ámbito normativo interno. Por ejemplo, la aplicación de la Ley 60 de 1993 sobre recursos y competencias.

El proceso de ejecución de la política pública implica, por lo general, la intervención de una cadena de actores administrativos, cuyo comportamiento va desagregando $y$, a la vez, materializando la política. Esto nos lleva a afirmar que pocas veces la política se implementa como se formula y que en los espacios regionales, con sus particulares características socioeconómicas y políticas, estas tienen diferentes puntos de concreción e, igualmente, disímiles resultados.

La ejecución de políticas involucra al sistema de poder institucional, que toma las decisiones; a la administración pública, encargada de ejecutarlas, y a la sociedad, que recibe los beneficios o perjuicios de la acción pública. Por ello, alrededor de una política y de su proceso de gestación, formulación, ejecución, evaluación, se interrelacionan el sistema de poder político, el aparato administrativo y la sociedad, $\mathrm{y}$ se tejen un conjunto de interacciones a su alrededor. 


\section{Factores que influyen en la política pública}

Las políticas públicas no sólo responden a intentos voluntaristas del régimen político o de los distintos actores sociales. También se pueden ubicar en contextos específicos dentro de los cuales los de tipo estructural inciden con gran peso sobre el tipo de políticas que finalmente formulan los Estados y sobre su implementación.

La política debe entenderse dentro de un contexto internacional determinado así, en la medida en que se globaliza la economía, la política y el derecho. Èstos aspectos inciden sobre las políticas de los Estados nacionales. Cada vez es más difícil pensar una política pública que haga caso omiso del contexto internacional en que se sitúa. En este sentido es más frecuente frente a situaciones problemáticas en las cuales se pasa crecientemente de un concepto de soberanía nacional clásico de los Estados nacionales a uno de soberanías compartidas entre varios Estados o impulsados por organismos internacionales como la Organización Internacional del Trabajo (OIT), la Organización Mundial de la Salud (OMS) y las Naciones Unidas (Parra, 2002).

La política y el derecho internacional, en virtud de la severa discriminación y vulneración de los derechos humanos relacionados con la población con discapacidad, han generado una política global supraestatal mediante diversos tratados regionales. Por ejemplo el Tratado de Ámsterdam en Europa y la Convención Interamericana para la Eliminación de todas las formas de Discriminación contra las Personas con Discapacidad.

En este mismo sentido, observamos cómo el sistema de Naciones Unidas ha presentado una evolución en torno a la protección del colectivo de personas con discapacidad desde los años setenta, pues ha pasado por una serie de declaraciones hasta llegar a las normas uniformes para la eliminación de barreras para las personas con discapacidad. En esta época la tendencia en la formulación de políticas se ha centrado en el reconocimiento de las diferencias de las personas con discapacidad, como una condición del ser humano. También se ha enfocado en la promoción de procesos y actividades para mejorar las oportunidades y el desarrollo de la autonomía personal, la integración y participación en la vida cotidiana, la familia, la comunidad y la sociedad en general.

Como principios generales de la política global en discapacidad se pueden establecer por lo menos cuatro: igualdad de oportunidades, accesibilidad a los servicios, no discriminación y autonomía personal. Estos elementos fundamentales han sido establecidos en la Declaración de los Derechos de los Impedidos (ONU, 1975), Normas Uniformes sobre Igualdad de Oportunidades para las Personas con Discapacidad (ONU, 1996) y Programas de Acción Mundial para los Impedidos (ONU, 1982).En síntesis corresponden a las siguientes formulaciones:

- Igualdad de oportunidades: concebido como el principio que reconoce la importancia de las diversas necesidades del individuo, que deben constituir la base de la planificación de la sociedad. Tienen como fin de asegurar el empleo de los recursos para garantizar que las personas disfruten de iguales oportunidades, de acceso y participación en idénticas circunstancias.

- Accesibilidad: ausencia de restricciones para que todas las personas puedan movilizarse libremente en el entorno, hacer uso de todos los servicios requeridos y disponer de todos los recursos que garanticen su seguridad, movilidad y comunicación. ${ }^{4}$

- No discriminación: la no discriminación implica no excluir, restringir, impedir o anular el reconocimiento, goce o ejercicio de los derechos humanos o libertades fundamentales a una persona. $^{5}$

- Participación de las personas con discapacidad: derecho de las personas con discapacidad de intervenir en la toma de decisiones, planificación, ejecución y control de las acciones que los involucran.

- Autonomía: derecho de las personas con discapacidad de tomar sus propias decisiones y 
el control de las acciones que las involucran para una mejor calidad de vida, basada dentro de lo posible en la autosuficiencia (Castañeda y Parra, 2003, pp. 351-372).

\section{Las políticas públicas condicionadas por el régimen político}

En este orden de ideas las políticas públicas se ven condicionadas por el tipo de régimen político. Esta restricción posibilita o no la expresión y movilización de los diversos actores políticos y sociales frente al problema, demanda o necesidad que motiva la política, con lo que pueden privilegiar bien sea las salidas consensuales o bien las de tipo impositivo. Por lo tanto, podemos afirmar que la política estatal está condicionada por un juego de fuerzas dentro de las cuales podemos destacar, además de las dos señaladas, las siguientes:

En primer lugar, las características, el tamaño, la complejidad y la ubicación del problema, demanda o necesidad que origina la política. Así las cosas, encontramos que la discapacidad en Colombia, como problemática social, no se puede ubicar en un punto de localización específico del país, sino indiscriminadamente en el territorio nacional con la complejidad que implica el conjunto de necesidades del individuo. Las carencias de estos sujetos van desde aspectos de asistencia social hasta sus intereses de participación sociopolítica, sin perder de vista el sentido de integridad de estas personas. Situaciones de dificultoso análisis para los tecnócratas y, en ocasiones, para la misma población que demanda. De ahí la dificultad de formular e implementar políticas públicas para esta población con un efectivo y real impacto social.

En segundo lugar, el proyecto político del gobierno, en cierta forma, condiciona el tipo de respuesta y, en alguna medida, se expresa en el plan de desarrollo. Por esta razón lo que es difícil pensar una política pública específica que vaya en contravía de lo que podemos denominar las megapolíticas o políticas básicas de cada momento.
En la última década, basándose en las oportunidades y garantías que genera la Constitución de 1991 y dada una mayor organización de las personas con discapacidad, esta población se convirtió en una fuerza sociopolítica de presión, que hoy incide en la formulación de políticas públicas encaminadas a resolver sus necesidades como consecuencia de su vulnerabilidad. ${ }^{6}$

En tal medida encontramos que la política pública en Colombia, aplicada a la discapacidad, ha trascendido (con poca incidencia) de los ámbitos territoriales al plan de desarrollo nacional, como elemento esencial para formular y aplicar las tan mencionadas políticas. Esto si se sigue el sinuoso recorrido desde la formulación, que se basa en la participación regional, en su consolidación nacional y en su aplicación nuevamente a las regiones. La razón es que el acceso a los ámbitos de participación regional implican un menor volumen de personas y logran un mayor impacto e inmediatez como resultado de su gestión.

No se puede desconocer que parte del problema puede estar motivado por la falta de organización. La estructura nacional de las personas con discapacidad, que les permita convertirse en un grupo de presión claramente identificado. Por lo tanto, que pueda incidir en el diseño, en la formulación y en la implementación de las políticas públicas en los marcos programáticos de las administraciones respectivas, en sus diferentes ámbitos de gobierno (nación, departamento y municipio).

En tercer lugar, la sociedad civil organizada y representada en organizaciones de personas con discapacidad o para éstas, al no percibir tanto una política pública para el sector como unos resultados efectivos a sus necesidades en los sectores de educación, salud, trabajo y comunicaciones (Castañeda, 1998). La sociedad civil ha generado diversas respuestas a las problemáticas que se le han presentando. A partir de aquí sus procesos de efectividad como sociedad se ven afectados por los siguientes factores: 
Las problemáticas para la discapacidad varían de acuerdo con el tipo en términos de dar prioridad a los problemas, toda vez que el acceso a la integración educativa de las personas sordas, no es el misma que el de las personas con otra clase de limitación física. Lo anterior está condicionado al desarrollo técnico y tecnológico del sistema educativo para asumir el proceso de integración educativa, que restringe significativamente a las personas con limitación auditiva (Castañeda y Parra, 2003).

En el ámbito nacional, respecto a las estrategias de solución de problemas por falta de una estructura dentro de la respectiva discapacidad o entre los grupos de discapacidad (físicas, psíquicas y sensoriales), sus acciones no tienen un impacto global. Es así como las gestiones son locales y, como ya se anotó anteriormente, se realizan por la organización de personas con discapacidad o para ellas. Este proceso se lleva a cabo de manera aislada con respecto a sus organizaciones homologas de carácter nacional o regional.

En el ámbito político se han generado dos tipos de acciones. Por un lado, las de las personas con discapacidad que personalmente han intentado vincularse como representantes a los consejos, la Asamblea o el Congreso, con el fin de intentar desde el ámbito legislativo intervenir positivamente en la normativa referida a la discapacidad. Por otro, en forma más organizada, los líderes, en representación de los diferentes grupos de personas con discapacidad, se han vinculado en forma directa a las estructuras de los partidos o movimientos, con el fin de involucrar en sus plataformas los temas relacionados con las políticas públicas que se van a desarrollar en beneficio a esta población. ${ }^{7}$

La disponibilidad y el gasto de recursos estatales obran casi siempre como factores condicionantes, que tiene incidencias sobre la política. Estos aspectos se denotan en nuestro país debido a la deuda externa, al gasto militar, a la tendencia negativa en el crecimiento económico, e influyen en los presupuestos de inversión social. Por eso, aquellos que posiblemente pudieran ser asignados a las políticas públicas orientados a las personas con discapacidad se diluyen en programas puntuales con bajo impacto social (Vargas, 1995, pp. 55-65).

En el ámbito regional se ha encontrado mayor asignación presupuestal para los programas o proyectos de beneficio a las personas con discapacidad, debido tanto la participación de éstos en la elaboración de los planes de desarrollo como a la sensibilidad y voluntad política de las autoridades municipales o departamentales.

Lo anterior no pretende colocar en un mismo pie de igualdad a factores desiguales, ya sea por su capacidad de presión o por las posibilidades de dar prioridad a sus intereses. La fuerza determinante en la definición de la política estatal, en el ámbito interno, la da el proyecto político dominante en cada momento histórico, que por momentos asimila parcialmente las propuestas alternativas de otros actores, siempre y cuando no sean contradictorias con sus intereses. O bien cuando requiere conciliarse con éstos para efectos de ganar un mayor consenso alrededor de sus políticas.

Por último, es importante aclarar que las dimensiones espacial y temporal en que se ubican las políticas públicas pueden afectar sus resultados. Esto se explica porque adquieren diferentes expresiones en distintos momentos históricos o en diversos espacios nacionales y regionales, por las diferentes configuraciones de cada región en lo económico, lo político, lo cultural y lo administrativo-.

\section{Reformulación de la política pública en discapacidad}

A continuación establecemos algunos aspectos generales que pueden ser motivo para la reformulación de las políticas públicas en discapacidad. Igualmente planteamos algunas propuestas de política a partir de la valoración 
de documentos como Plan Nacional de Atención para Personas con Discapacidad 1998-2002; la evaluación de la Ley 361 de 1997, realizada por la Consejería Social, Presidencia de la República, en el 2001, y el estudio realizado por la Universidad del Rosario Realidad social de la población con discapacidad en Colombia.

El marco constitucional, las leyes, los decretos reglamentarios y, en general, la normatividad que se deriva de los ámbitos departamental y local suelen discriminar las obligaciones de un sector y no su coordinación con los otros; ya que no tienen en cuenta las necesidades de las personas con discapacidad que, en términos de los servicios considerados como integrales para efectos de su prestación, no incluyen un trabajo de diversos sectores, interdisciplinario que abarque los distintos aspectos del ser humano.

Así mismo, dicho marco normativo, en la mayoría de ocasiones, no establece los aspectos presupuestales o los de vigilancia, inspección y control. En consecuencia se generan dificultades de competencia $\mathrm{y}$, por ende, déficit en la prestación de servicios. Así se constituyen entidades con determinadas competencias que en la práctica no se realizan.

Otra variable entre tantas que se suscitan y afectan la adecuada implementación de la política pública, es la mayor o menor dependencia de las mismas derivada de la cooperación internacional. La explicación está en que muchos de los planes que se adelantan y ponen en marcha terminan abruptamente debido a la escasez en los recursos económicos con los que se inició, lo que lo hace poco sostenible. Debido a ello, en algunos casos se cuenta buenos planes de acción en este orden y desafortunadamente se paraliza la gestión ocasionando en algunos casos expectativas de derechos y signos de progreso de manera inconclusa. La mejor solución para este tipo de problemáticas al nivel de la puesta en marcha de la política pública, es la apropiación de recursos públicos que permitan la consecución de los objetivos, sin que se dependa en exclusiva de los ingresos percibidos por organizaciones no gubernamentales y entes internacionales.

Por otra parte, la falta de claridad conceptual, por parte de funcionarios públicos o privados en el manejo de algunos de los términos en la interpretación de modelos de atención que asisten o prestan servicios a población con discapacidad, incide directamente en ella. Sucede que en algunos casos estas interpretaciones no corresponden a sus necesidades. La razón es que se hacen de manera aislada al proceso de incorporación social o simplemente no se les presta atención por no considerarlos personas beneficiarias de un programa de atención en salud, educación, trabajo, etc; quienes los diseñan no incluyen las actividades específicas de acuerdo con sus necesidades.

\section{La política pública en discapacidad y los} derechos humanos en Colombia

El enfoque en derechos humanos de la política pública es un ejercicio relativamente reciente en el Estado colombiano. En realidad se podría hablar de una aproximación a esta tendencia a partir de la normatividad que pretendía dar un poco de aliento a la crítica situación de la población desplazada en nuestro país. Sin embargo el desarrollo de esta perspectiva ha sido marginal en los planes de gobierno a través de los años. Con el fin de clarificar este punto se puede citar el Plan de Desarrollo Distrital de la ciudad de Bogotá, más conocido como "Bogotá sin indiferencia", que está por concluir. En él, la inclinación central para la construcción de la política pública es la concepción de los derechos humanos, a partir de la interdependencia de los mismos. Este ejemplo quizá lo más cercano a la creación de la cultura en derechos humanos, a través de la construcción de la política pública.

De acuerdo con lo anterior puede afirmarse que las realidades del contexto social, económico y político, al igual que los compromisos de carácter internacional adquiridos por Colombia obligan a realizar un esfuerzo de comprensión entre las 
políticas públicas locales, las tendencias mundiales en el desarrollo y la obligación del cumplimiento de convenios y tratados sobre derechos humanos y derecho internacional humanitario, con el propósito de tener una visión más integral en el abordaje y solución a los problemas que aquejan nuestro país.

El conflicto armado, los problemas humanitarios, la pobreza y el hambre, la inequidad, el limitado crecimiento económico, el déficit en el acceso a los servicios de salud, educación, vivienda así como la insuficiencia en la atención a los derechos de los grupos vulnerables como infancia, mujer, tercera edad, personas con discapacidad, indígenas y afrocolombianos representan el reto de diseñar políticas públicas articuladas que orienten nuevas visiones del desarrollo en una perspectiva de derechos en el marco de un proceso de concertación entre el Gobierno y la sociedad civil.

En términos de los derechos humanos y el derecho internacional humanitario, el país ha suscrito una serie de convenios y tratados internacionales que obligan al Estado a la adopción de una serie de decisiones políticas, administrativas y jurídicas que permitan la efectiva protección a los derechos humanos. Pero que al tiempo viabilicen procesos de paz, así como la atención efectiva a las víctimas del conflicto armado, dentro de un marco de verdad, justicia y reparación.

De otra parte, Colombia, al suscribir la Declaración y el Plan de Acción de la Conferencia Mundial de Derechos Humanos, adquirió el compromiso de elaborar un Plan Nacional de Acción en Derechos Humanos, compromiso que fue ratificado en el Plan Nacional de Desarrollo

\section{Hacia un Estado comunitario.}

Igualmente, en términos del desarrollo, Colombia suscribió la Declaración del Milenio que obliga al cumplimiento de unos objetivos de desarrollo del milenio en un período de tiempo determinado, para lo cual se han adoptado otra serie de decisiones de política pública. Es el caso del Plan de Desarrollo "Hacia un Estado comunitario", el Conpes 091 de 2003, las siete herramientas de equidad social, la misión contra la pobreza y la agenda interna de productividad y competitividad.

Tradicionalmente, los temas de los derechos humanos y el desarrollo humano se han tratado de forma independiente y desarticulada. Generalmente al primero se le vincula con los derechos civiles y políticos del individuo y al segundo con problemas sociales y colectivos.

Sin embargo, las últimas teorías del desarrollo humano y los derechos humanos, si bien con sus particularidades, se complementan entre sí. En palabras de Sen, "el desarrollo humano y los derechos humanos tienen una motivación común: "La idea del desarrollo humano se centra directamente en el progreso de la vida y el bienestar humanos". Como el bienestar incluye vivir con libertades sustanciales, el desarrollo humano también está vinculado integralmente al fortalecimiento de determinadas capacidades relacionadas con toda la gama de cosas que una persona puede ser y hacer en su vida. Valoramos la libertad de poder vivir como nos gustaría hacerlo, e incluso la oportunidad de escoger nuestra propia suerte" (PNUD, 2003).

Así mismo, los dos enfoques están en capacidad de aportar y enriquecerse entre sí en particular, frente a los vínculos de los derechos humanos con los Objetivos de Desarrollo del Milenio, se ha señalado que:

"Los derechos humanos y el desarrollo humano están íntimamente unidos y forman parte de la esencia de los Objetivos de Desarrollo del Milenio [...]. La mejor manera de alcanzar cada uno de estos Objetivos consiste en abordar toda la problemática relacionada con los derechos humanos: la igualdad, la no discriminación, la participación, la 
inclusión, la rendición de cuentas y la justicia social, además de la solidaridad y la cooperación internacionales. Ello quiere decir que, para conseguir los ODM, debemos basar nuestra actuación en los derechos humanos, prestando atención no sólo a los resultados del desarrollo sino también a las formas de luchar por el desarrollo" (Malloch en PNUD, 2005)".

"Las normas de derechos humanos brindan un marco para la igualdad y la no discriminación que, aplicado de manera objetiva, asegura que los beneficios del desarrollo humano alcancen incluso a las personas más marginadas. Es más, dichas normas agregan al enfoque de eficiencia y provisión de servicios el aspecto crucial de la legitimidad" (PNUD, 2005).

En consonancia con la tesis anterior, es pertinente y necesario hacer visible la articulación entre las bases del Plan de Acción de Derechos Humanos y Derecho Internacional Humanitario y los Objetivos de Desarrollo del Milenio para garantizar una armonía que permita abordar el desarrollo humano desde la perspectiva de los derechos.

\section{Conclusiones}

Así pues, podemos concluir, que el fin último de la política pública social es expandir la ciudadanía, la democracia radical, la igualdad y la inclusión, el respeto a los derechos humanos y las libertades públicas generando espacios y garantías para que las personas y las comunidades puedan interactuar y elegir autónomamente sus propios destinos y planes de vida. A partir de allí, podemos preguntarnos, qué política publica realmente queremos.

En su lugar es necesario que se elaboren permanentes evaluaciones a las políticas adelantadas e implementadas, con el objeto de crear un constante ejercicio de retroalimentación de la política que permita darle giros o modificaciones cuando esto se torne necesario y así ser consecuentes con la dinámica que va marcando la realidad de la sociedad.

Resulta pertinente así mismo que se encuentren identificados los enfoques deseados en la política, que se cree la conciencia de sostenibilidad de las mismas a partir de la designación de apropiaciones presupuestales que sean la base de su desarrollo, quedando así la cooperación internacional como un refuerzo a avance de las mismas. Este proceso garantiza que no se paralice en los eventos en los que se agotan los recursos económicos.

Se hace imperativo así mismo vincular el desarrollo humano con los derechos humanos, en aras a que a partir de la comunicación efectiva entre estos dos aspectos se mejore la gestión de los recursos y se logren mejores finalidades en el desarrollo y progreso de las políticas con enfoque social.

La formulación de las políticas sociales, y en especial las dedicadas a dar tratamiento al tema de la discapacidad, deben ser construidas a la luz de los objetivos del milenio y atendiendo igualmente a los numerosos instrumentos internacionales suscritos por el Estado Colombiano en esta materia. Toda vez que solo a partir de este dialogo entre instancias internas e internacionales se puede caminar hacia el mejoramiento de las condiciones de vida de los individuos y la lucha contra la discriminación y de la exclusión.

\section{Notas}

${ }^{1}$ Resulta ser por tanto el perfecto ejemplo del sano ejercicio de la construcción de la política pública, en el sentido en el que se diseña en un primer momento, pero luego de una evaluación de ciertas medidas puntuales es objeto de reformulación.

${ }^{2}$ Estos conceptos de Discapacidad y el anterior de MSR, han sido desarrollados tanto en el documento Conpes 080 de discapacidad como en el libro de "Bases para la Formación de Política Pública en Discapacidad", razón por la 
cual no se les da un mayor desarrollo en el presente plan.

${ }^{3}$ CIF: Clasificación Internacional del Funcionamiento, la Discapacidad y la Salud. OMS. Mayo de 2001.

${ }^{4} \mathrm{El}$ acceso a la comunicación tanto para las personas con limitación visual como para las personas con limitaciones físicas graves y sordas, entre otros aspectos, implica el acceso mediante tecnologías de punta a sistemas computarizados, internet, sistemas de traducciones en subtítulos (close-caption), entre otros.

${ }^{5}$ Recordemos que en Colombia la Convención Interamericana para la Eliminación de todas las Formas de Discriminación contra las Personas con Discapacidad fue ratificada por Colombia con la ley 762 de 2003.en

Esta Convención trata los distintos ámbitos de la discriminación, como la distinción, la exclusión o la restricción basada en la discapacidad. Artículo I, numeral 2.

${ }^{6}$ Colombia con la Constitución de 1991 se declara como un Estado social de derecho, con lo cual prevalecen nuevos valores como el pluralismo, democracia participativa y moral social. Asimismo, los fundamentos de este nuevo Estado son el respeto a la dignidad humana, el trabajo, la solidaridad y la prevalencia del interés general.

${ }^{7}$ Como crítica podemos observar que los distintos partidos políticos, en la precampaña, han recogido las expectativas de la población con discapacidad más como estrategia política, en busca de este posible caudal electoral, que por un verdadero interés de desarrollar una política pública seria de la discapacidad.

\section{Referencias}

Castañeda, H. y Parra Dussan, C. A. (2003), Estado de crisis o crisis del estado, el estado y la realidad de la política pública en discapacidad, Bogotá, Centro editorial Universidad del rosario.
Castañeda, H. (1998), Integración educativa de las personas con discapacidad, [tesis de maestría], Bogotá, Pontificia Universidad Javeriana, Maestría en Estudios Políticos y Relaciones.

Gracas Rúa M. (2002), en Molina, C. G. Modelo de Formación de Políticas y Programas Sociales, Instituto Interamericano para el Desarrollo Social (INDES)

Malloch Brown, M. (2005), en PNUD, Los Derechos Humanos en el PNUD.

Naciones Unidas, Asamblea General (1993, 20 de diciembre), Resolución 48/96.

(1982, 3 de diciembre), Resolución 37/52.

(1975, 9 de diciembre), Resolución 3447.

Parra Dussan, C. (2002), La protección jurídico internacional de la población con discapacidad, Bogotá, Universidad del Rosario.

Ley 812de 2003, Plan de Desarrollo Nacional, diario oficial 45.231, Bogotá, junio, 2003.

Plan Nacional de Atención para las Personas con Discapacidad 1999-2002, Imprenta Nacional, Bogotá, 1999.

PNUD (2000), Informe sobre Desarrollo Humano.

Presidencia de la república, CPPE - RSS (2002), Bases para la Formación de Politica Pública en Discapacidad, Bogotá.

Sarmiento Anzola, L. (2005, 4 y 5 de febrero), "Las Políticas Públicas y el Desarrollo Local" [conferencia], Bogotá.

Vargas Velásquez, A. (1995), Notas sobre el estado y las políticas públicas, Almudena, s. L. 